Rev. Mat. Iberoamericana 19 (2003), 57-72

\title{
Complex geometrical optics solutions for Lipschitz conductivities
}

\section{Lassi Päivärinta, Alexander Panchenko and Gunther Uhlmann}

\begin{abstract}
We prove the existence of complex geometrical optics solutions for Lipschitz conductivities. Moreover we show that, in dimensions $n \geq 3$ that one can uniquely recover a $W^{3 / 2, \infty}$ conductivity from its associated Dirichlet-to-Neumann map or voltage to current map.
\end{abstract}

\section{Introduction}

Let $\Omega \subset \mathbb{R}^{n}$ be a bounded domain with Lipschitz boundary. Let $\gamma \in L^{\infty}(\Omega)$ be the electrical conductivity of $\Omega$. We assume throughout the paper that the conductivity is strictly positive on $\bar{\Omega}$. Given a voltage potential $f$ on $\partial \Omega$, under the assumption of no sources or sinks of current on $\Omega$, the induced potential $u$ on $\Omega$ satisfies the Dirichlet problem

$$
\begin{aligned}
\operatorname{div}(\gamma \nabla u) & =0 \quad \text { on } \quad \Omega \\
u_{\left.\right|_{\partial \Omega}} & =f .
\end{aligned}
$$

The Dirichlet-to-Neumann map (DN), or voltage to current map, is defined by

$$
\Lambda_{\gamma}(f)=\left(\gamma \frac{\partial u}{\partial \nu}\right)_{\partial \Omega}
$$

where $u$ is a solution to $(1.1)$ and $\nu$ denotes the unit outer normal to $\partial \Omega$.

2000 Mathematics Subject Classification: 35R30 35Q60.

Keywords: Electrical Impedance Tomography, Complex Geometrical Optics, Lipschitz Conductivities. 
The inverse problem of determining $\gamma$ from $\Lambda_{\gamma}$ has been extensively studied since Calderón's pioneer paper [4]. The essential tool for the solution is the construction of complex geometrical optics solutions for the underlying conductivity equation. See [16] for a recent survey.

Kohn and Vogelius proved that one can uniquely determine piecewise real-analytic conductivities from the DN map [7]. Sylvester and Uhlmann proved in dimension $n \geq 3$ a global identifiability result for smooth conductivities [13]. Brown extended this result to conductivities in $C^{3 / 2+\varepsilon}(\bar{\Omega})$ for any $\varepsilon>0$, [2]. In two dimensions Nachman proved a global identifiability result for conductivities having two derivatives [8]. This was improved to Lipschitz conductivities in [3]. We point out that the above inverse conductivity problem makes sense for conductivities that are only in $L^{\infty}$. There are neither proofs nor counter-examples for this case in any dimension.

As mentioned above a crucial ingredient in the proof of the results in [2], $[3],[8]$, and [13] is the construction of complex geometrical optics solutions for the equation (1.1), [13, 12].

Let $\rho \in \mathbb{C}^{n}$ with $\rho \cdot \rho=0$. Moreover, let $\gamma \in C^{2}\left(\mathbb{R}^{n}\right), n \geq 2$, with $\gamma=1$ for $|x| \geq R$ and $R$ sufficiently large. Then for $|\rho|$ sufficiently large there exist solutions of $\operatorname{div}(\gamma \nabla u)=0$ in $\mathbb{R}^{n}$ of the form

$$
u=e^{x \cdot \rho} \gamma^{-1 / 2}\left(1+\psi_{\gamma}(x, \rho)\right) .
$$

In two dimensions these solutions are constructed for all $\rho \in \mathbb{C}^{2} \backslash\{0\}$ with $\rho \cdot \rho=0,[8]$.

The function $\psi_{\gamma}$ satisfies

$$
(\Delta+2 \rho \cdot \nabla) \psi_{\gamma}=q\left(1+\psi_{\gamma}\right)
$$

where

$$
q=\frac{\Delta \sqrt{\gamma}}{\sqrt{\gamma}}
$$

An important property of $\psi_{\gamma}$ is that

$$
\psi_{\gamma} \stackrel{|\rho| \rightarrow \infty}{\longrightarrow} 0
$$

in an appropriate sense so that the solutions of (1.4) behave like $e^{x \cdot \rho} \gamma^{-1 / 2}$ for large $|\rho|$.

In this paper we construct complex geometrical optics solutions for conductivities $\gamma \in W^{1, \infty}\left(\mathbb{R}^{n}\right)$. We sketch some of the steps in the construction. 
We first rewrite (1.1) in the form

$$
\Delta u+A \cdot \nabla u=0
$$

where $A=\nabla \log \gamma \in L^{\infty}\left(\mathbb{R}^{n}\right)$ has compact support. Let $A_{\varepsilon}=A * \Phi_{\varepsilon}$ where $\Phi_{\varepsilon} \in C_{0}^{\infty}\left(\mathbb{R}^{n}\right), \varepsilon>0$, is an approximation to the $\delta$-function.

We consider instead of (1.6) the Schrödinger equation with a smooth first order term

$$
\Delta u_{\varepsilon}+A_{\varepsilon} \cdot \nabla u_{\varepsilon}=0
$$

In [9] complex geometrical optics solutions to (1.7) are constructed of the form

$$
u_{\varepsilon}=e^{x \cdot \rho} e^{-\frac{\varphi_{\varepsilon}(x)}{2}} r_{\varepsilon}(x, \rho),
$$

with $r_{\varepsilon}$ satisfying appropriate decay conditions as $|\rho| \rightarrow \infty$. The functions $\varphi_{\varepsilon}$ are defined by

$$
\varphi_{\varepsilon}=\varphi * \Phi_{\varepsilon}
$$

We remark that in the case that $A_{\varepsilon}=\nabla \log \alpha$ with $\alpha$ smooth, then

$$
e^{-\frac{\varphi_{\varepsilon}(x)}{2}}=\alpha^{-1 / 2} \text {. }
$$

Our complex geometrical optics solutions are of the form

$$
u=e^{x \cdot \rho}\left(\omega_{0}(x, \varepsilon)+\omega_{1}(x, \varepsilon, \rho)\right),
$$

with

$$
\omega_{0}(x, \varepsilon)=e^{-\frac{\varphi_{\varepsilon}(x)}{2}} .
$$

In Section 2 we see that $\omega_{0} \rightarrow \gamma^{-1 / 2}$ as $\varepsilon \rightarrow 0$, in appropriate sense. Since $\omega_{0}$ is smooth we have avoided the problem of taking two derivatives of $\gamma$.

Now $\omega_{1}$ solves the equation

$$
((\Delta+2 \rho \cdot \nabla)+A \cdot(\nabla+\rho)) \omega_{1}=-((\Delta+2 \rho \cdot \nabla)+A \cdot(\nabla+\rho)) \omega_{0} .
$$

We show that $\omega_{1} \rightarrow 0$ as $\varepsilon \rightarrow 0,|\rho| \rightarrow \infty$ in appropriate sense.

In this paper we show the existence of the complex geometrical solutions for all Lipschitz conductivities and give a global identifiability result for $W^{3 / 2, \infty}$ conductivities in dimension three and higher. This latter result improves on the result of [2]. 
More precisely, we will prove:

Theorem 1.1 Let $\gamma \in W^{1, \infty}\left(\mathbb{R}^{n}\right)$, $\gamma$ strictly positive and $\gamma=1$ outside a large ball. Let $-1<\delta<0$. Then for $|\rho|$ sufficiently large there is a unique solution of

$$
\operatorname{div}(\gamma \nabla \mathrm{u})=0 \quad \text { in } \quad \mathbb{R}^{\mathrm{n}}
$$

of the form

$$
u=e^{x \cdot \rho}\left(\gamma^{-1 / 2}+\psi_{\gamma}(x, \rho)\right)
$$

with $\psi_{\gamma} \in L_{\delta}^{2}\left(\mathbb{R}^{n}\right)$.

Moreover, $\psi_{\gamma}$ has the form

$$
\psi_{\gamma}(x, \rho)=\left(\omega_{0}(x, \rho)-\gamma^{-1 / 2}\right)+\omega_{1}(x, \rho)
$$

where $\omega_{0}$ and $\omega_{1}$ satisfy

$$
\lim _{|\rho| \rightarrow \infty}\left\|\omega_{0}(x, \rho)-\gamma^{-1 / 2}\right\|_{H_{\delta}^{1}}=0
$$

and

$$
\lim _{|\rho| \rightarrow \infty}\left\|\omega_{1}(x, \rho)\right\|_{L_{\delta}^{2}}=0
$$

Theorem 1.2 Let $n \geq 3$. Let $\gamma_{i} \in W^{3 / 2, \infty}(\Omega)$, be strictly positive on $\bar{\Omega}$, $i=1,2$. Assume $\Lambda_{\gamma_{1}}=\Lambda_{\gamma_{2}}$. Then $\gamma_{1}=\gamma_{2}$ on $\Omega$.

Note that if one could replace $L_{\delta}^{2}$-norm in (1.15) with $H_{\delta}^{1}$-norm then Theorem 1.2 would easily follow for any $W^{1, \infty}(\Omega)$ conductivity.

In Section 2 we construct solutions as in Theorem 1.1. The key point of the proof is the observation that the operator on the left-hand side of (1.11) has an explicit approximative inverse that involves only first order derivatives of $\gamma$ (c.f. Theorem 2.1 below).

In Section 3 we prove Theorem 1.2, using a new identity that is satisfied if the DN maps of two conductivities are the same and plugging the solutions (1.9) into this identity. The standard identity used in previous results doesn't seem to give the desired result.

As for the case of $W^{2, \infty}(\Omega)$ conductivities, we expect that the methods of this paper will lead to stability estimates and a reconstruction method. The case of $C^{2 / 3+\varepsilon}(\bar{\Omega}), \varepsilon>0$, first order perturbations of the Laplacian was considered in [15]. 


\section{Construction of the complex geometrical optics so- lutions}

In this section we construct $\omega_{0}$ and $\omega_{1}$ as in Theorem 1.1 and we derive their asymptotic properties as $|\rho| \rightarrow \infty$.

We denote by $\Phi_{\varepsilon}(x)=\varepsilon^{-n} \Phi(x / \varepsilon), \varepsilon>0$, an approximation to the $\delta$-function. More specifically we assume

$$
\Phi(x)=\prod_{j=1}^{n} \Phi_{0}\left(x_{j}\right)
$$

with $\Phi_{0} \in C_{0}^{\infty}(\mathbb{R}), \Phi_{0} \geq 0, \Phi_{0}=1$ near 0 and $\int \Phi_{0}(x) d x=1$.

We define the weighted $L^{2}$-space, $L_{\alpha}^{2}$, by

$$
L_{\alpha}^{2}\left(\mathbb{R}^{n}\right)=\left\{f: \int\left(1+|x|^{2}\right)^{\alpha / 2}|f(x)|^{2} d x<\infty\right\}
$$

with norm given by

$$
\|f\|_{L_{\alpha}^{2}}^{2}=\int\left(1+|x|^{2}\right)^{\alpha}|f(x)|^{2} d x
$$

We denote by $H_{\alpha}^{s}\left(\mathbb{R}^{n}\right)$ the corresponding Sobolev space defined by interpolation for non integer $s$.

Throughout this section we assume that $\gamma \in W^{1, \infty}\left(\mathbb{R}^{n}\right)$, with $\gamma=1$ outside a large ball. Let

$$
\begin{aligned}
\varphi_{\varepsilon} & =\Phi_{\varepsilon} * \log \gamma, \\
A & =\nabla \log \gamma, \\
A_{\varepsilon} & =\Phi_{\varepsilon} * A .
\end{aligned}
$$

Clearly

$$
\rho \cdot \nabla \varphi_{\varepsilon}=\rho \cdot A_{\varepsilon}
$$

Next we define

$$
\omega_{0}(x, \varepsilon)=e^{-\varphi_{\varepsilon}(x) / 2}
$$


62 L. Päivärinta, A. Panchenko and G. Uhlmann

The proof of the following proposition is standard.

Proposition 2.1 If $A \in W^{1 / 2, \infty}(\Omega)$ we have as $\varepsilon \rightarrow 0$

$$
\left\|A_{\varepsilon}-A\right\|_{L^{2}}=\varepsilon^{1 / 2} O(\varepsilon)
$$

and

$$
\left\|\partial_{x_{j}} A_{\varepsilon}\right\|_{L^{2}}=\varepsilon^{-1 / 2} O(\varepsilon) .
$$

From the above proposition we conclude

Lemma 2.1 Let $\omega_{0}$ be as in (2.5). Then for any $\delta>0$

$$
\lim _{\varepsilon \rightarrow 0}\left\|\omega_{0}-\gamma^{-1 / 2}\right\|_{H_{\delta}^{1}}=0
$$

Let $\rho \in \mathbb{C}^{n}$ with $\rho \cdot \rho=0$. We define the operators

$$
\begin{gathered}
\Delta_{\rho} u=e^{-x \cdot \rho} \Delta\left(e^{x \cdot \rho} u\right)=\Delta u+2 \rho \cdot \nabla u, \\
\nabla_{\rho} v=e^{-x \cdot \rho} \nabla\left(e^{x \cdot \rho} u\right)=\nabla v+\rho \cdot v .
\end{gathered}
$$

Let $f \in C_{0}^{\infty}\left(\mathbb{R}^{n}\right)$. We define

$$
\Delta_{\rho}^{-1} f=\frac{1}{(2 \pi)^{n}} \int e^{i x \cdot \xi} \frac{\hat{f}(\xi)}{-|\xi|^{2}+2 i \rho \cdot \xi} d \xi
$$

and recall

Proposition 2.2 ([13, 12]). Let $n \geq 2$. Let $-1<\delta<0$ and $s \geq 0$. Then $\Delta_{\rho}^{-1}$ extends to a bounded operator

$$
\Delta_{\rho}^{-1}: H_{\delta+1}^{s}\left(\mathbb{R}^{n}\right) \rightarrow H_{\delta}^{s}\left(\mathbb{R}^{n}\right)
$$

with

$$
\left\|\Delta_{\rho}^{-1}\right\|_{H_{\delta+1}^{s} \rightarrow H_{\delta}^{s}} \leq \frac{C(s, \delta, n)}{|\rho|},
$$

for some $C>0$. Moreover

$$
\Delta_{\rho}^{-1}: L_{\delta+1}^{2}\left(\mathbb{R}^{n}\right) \rightarrow H_{\delta}^{k}\left(\mathbb{R}^{n}\right), \quad k=1,2
$$

is bounded and

$$
\left\|\Delta_{\rho}^{-1}\right\|_{L_{\delta+1}^{2} \rightarrow H_{\delta}^{k}} \leq C(\delta, k, n)|\rho|^{k-1}, \quad k=1,2,
$$

for some $C>0$. 
Next we construct $\omega_{1}$. To have a solution of the form (1.9), $\omega_{1}$ must satisfy

$$
\left(\Delta_{\rho}+A \cdot \nabla_{\rho}\right) \omega_{1}=-\left(\Delta_{\rho}+A \cdot \nabla_{\rho}\right) \omega_{0}=:-f_{\varepsilon}
$$

We write

$$
\omega_{1}=\Delta_{\rho}^{-1} \tilde{\omega}
$$

then $\tilde{\omega}$ must satisfy

$$
\left(I+A \cdot \nabla_{\rho} \Delta_{\rho}^{-1}\right) \tilde{\omega}=-f_{\varepsilon} .
$$

A straightforward calculation gives that

$$
f_{\varepsilon}=-e^{-\frac{\varphi_{\varepsilon}}{2}}\left(-\frac{1}{2} \Delta \varphi_{\varepsilon}+\frac{1}{4}\left(\nabla \varphi_{\varepsilon}\right)^{2}-\frac{1}{2} A \cdot \nabla \varphi_{\varepsilon}-\rho \cdot \nabla \varphi_{\varepsilon}+\rho \cdot A\right)
$$

Since $\rho \cdot \nabla \varphi_{\varepsilon}=\rho \cdot A_{\varepsilon}$, we get

$$
f_{\varepsilon}=-e^{-\frac{\varphi_{\varepsilon}}{2}}\left(-\frac{1}{2} \Delta \varphi_{\varepsilon}+\frac{1}{4}\left(\nabla \varphi_{\varepsilon}\right)^{2}-\frac{1}{2} A \cdot \nabla \varphi_{\varepsilon}+\left(A-A_{\varepsilon}\right) \cdot \rho\right) .
$$

In order to solve (2.12) we define the operators $T_{\rho}(\gamma)$ and $S_{\rho}(\gamma)$ by

$$
T_{\rho}(\gamma):=\left(I+A \cdot \nabla_{\rho} \Delta_{\rho}^{-1}\right)
$$

and

$$
S_{\rho}(\gamma):=\gamma^{-1 / 2}\left(I-A \cdot \nabla_{\rho} \Delta_{\rho}^{-1}\right) \gamma^{1 / 2}
$$

Note that

$$
S_{\rho}(\gamma)=\gamma^{-1 / 2} T_{\rho}\left(\gamma^{-1}\right) \gamma^{1 / 2}
$$

The main result of this section is that $T_{\rho}(\gamma)$ and $S_{\rho}(\gamma)$ are approximate inverses to each other. This result is used to prove that (2.12) has a unique solution in an appropriate space.

Theorem 2.1 Let $-1<\delta<0$ and $\gamma \in W^{1, \infty}\left(\mathbb{R}^{n}\right)$ with $\gamma$ strictly positive and $\gamma=1$ outside a large ball. Then the operator

$$
T_{\rho}(\gamma): L_{\delta+1}^{2}\left(\mathbb{R}^{n}\right) \rightarrow L_{\delta+1}^{2}\left(\mathbb{R}^{n}\right)
$$

is bounded and it has a bounded inverse $T_{\rho}^{-1}$ for $|\rho|$ large. Moreover

$$
\left\|T_{\rho}^{-1}(\gamma)-S_{\rho}(\gamma)\right\|_{L_{\delta+1}^{2} \rightarrow L_{\delta+1}^{2}} \rightarrow 0 \quad \text { as }|\rho| \rightarrow \infty
$$


64 L. PÄivärinta, A. Panchenko and G. Uhlmann

We first prove a stronger version of (2.19) for $C^{2}$-conductivities. More precisely we show

Lemma 2.2 Let $\gamma \in C^{2}\left(\mathbb{R}^{2}\right), \gamma=1$ outside a large ball and strictly positive. Then for $|\rho|$ large we have the estimate

$$
\left\|T_{\rho}^{-1}(\gamma)-S_{\rho}(\gamma)\right\|_{L_{\delta+1}^{2} \rightarrow L_{\delta+1}^{2}} \leq \frac{C\|\gamma\|_{C^{2}}}{|\rho|}
$$

for some $C>0$.

Proof. We have the following identity which was implicitly used in $[13,12]$ to reduce the construction complex geometrical optics solutions for the conductivity equation to the construction of complex geometrical optics solutions for the Schrödinger equation. The identity was explicitly stated in [5] and used to construct complex geometrical optics solutions for Maxwell's equations.

$$
\left(\Delta_{\rho}+A \cdot \nabla_{\rho}\right) \gamma^{-1 / 2}=\gamma^{-1 / 2}\left(\Delta_{\rho}-q\right)
$$

with $q=\frac{\Delta \sqrt{\gamma}}{\sqrt{\gamma}}$. Then

$$
\left(\Delta_{\rho}+A \cdot \nabla_{\rho}\right) \Delta_{\rho}^{-1}=\gamma^{-1 / 2}\left(\Delta_{\rho}-q\right) \gamma^{1 / 2} \Delta_{\rho}^{-1}
$$

and therefore

$$
I+\left(A \cdot \nabla_{\rho}\right) \Delta_{\rho}^{-1}=\gamma^{-1 / 2} \Delta_{\rho} \gamma^{1 / 2} \Delta_{\rho}^{-1}-q \Delta_{\rho}^{-1} .
$$

By Proposition 2.2

$$
\left\|q \Delta_{\rho}^{-1}\right\|_{L_{\delta+1}^{2} \rightarrow L_{\delta+1}^{2}} \leq \frac{C\|q\|_{\infty}}{|\rho|},
$$

for some $C>0$. Therefore

$$
\left(I+\left(A \cdot \nabla_{\rho}\right) \Delta_{\rho}^{-1}\right)^{-1}=\left(\gamma^{-1 / 2} \Delta_{\rho} \gamma^{1 / 2} \Delta_{\rho}^{-1}\right)^{-1}+B_{\rho},
$$

for $|\rho|$ large with

$$
\left\|B_{\rho}\right\|_{L_{\delta+1}^{2} \rightarrow L_{\delta+1}^{2}} \leq \frac{C\|q\|_{\infty}}{|\rho|}
$$

Since

$$
\left(\gamma^{-1 / 2} \Delta_{\rho} \gamma^{1 / 2} \Delta_{\rho}^{-1}\right)^{-1}=\Delta_{\rho} \gamma^{-1 / 2} \Delta_{\rho}^{-1} \gamma^{1 / 2}
$$

we conclude that

$$
\left\|\left(I+\left(A \cdot \nabla_{\rho}\right) \Delta_{\rho}^{-1}\right)^{-1}-\Delta_{\rho} \gamma^{-1 / 2} \Delta_{\rho}^{-1} \gamma^{1 / 2}\right\|_{L_{\delta+1}^{2} \rightarrow L_{\delta+1}^{2}} \leq \frac{C\|\gamma\|_{C^{2}}}{|\rho|} .
$$


Now we use formula (2.22) again by changing $\gamma$ to $1 / \gamma$, to conclude

$$
\Delta_{\rho} \gamma^{-1 / 2} \Delta_{\rho}^{-1} \gamma^{1 / 2}=\gamma^{1 / 2}\left(I-\left(A \cdot \nabla_{\rho}\right) \Delta_{\rho}^{-1}\right) \gamma^{-1 / 2}+\gamma^{1 / 2} \Delta\left(\gamma^{-1 / 2}\right) \Delta_{\rho}^{-1}
$$

From (2.23) and (2.24) we conclude the proof of the Lemma since by Proposition 2.2 again

$$
\left\|\gamma^{1 / 2} \Delta\left(\gamma^{-1 / 2}\right) \Delta_{\rho}^{-1}\right\|_{L_{\delta+1}^{2} \rightarrow L_{\delta+1}^{2}} \leq \frac{C\|\gamma\|_{C^{2}}}{|\rho|}
$$

Lemma 2.3 Let $\gamma \in W^{1, \infty}, \gamma$ strictly positive and $\gamma=1$ outside a large ball. Let $\gamma_{\varepsilon}=\gamma * \Phi_{\varepsilon}$. Then

$$
\left\|T_{\rho}\left(\gamma_{\varepsilon}\right)-T_{\rho}(\gamma)\right\|_{L_{\delta+1}^{2} \rightarrow L_{\delta+1}^{2}} \stackrel{\varepsilon \rightarrow 0}{\longrightarrow} 0
$$

Proof. We have that

$$
T_{\rho}\left(\gamma_{\varepsilon}\right)-T_{\rho}(\gamma)=I+\left(\nabla \log \gamma_{\varepsilon}-\nabla \log \gamma\right) \cdot \nabla_{\rho} \Delta_{\rho}^{-1} .
$$

Using Proposition 2.2 we conclude that

$$
\left\|\nabla_{\rho} \Delta_{\rho}^{-1}\right\|_{L_{\delta+1}^{2} \rightarrow L_{\delta+1}^{2}} \leq C
$$

for some $C>0$ independent of $|\rho|$. Therefore

$$
\left\|T_{\rho}\left(\gamma_{\varepsilon}\right)-T_{\rho}(\gamma)\right\|_{L_{\delta}^{2} \rightarrow L_{\delta+1}^{2}} \leq C\left\|\nabla \log \gamma_{\varepsilon}-\nabla \log \gamma\right\|_{L^{\infty}\left(\mathbb{R}^{n}\right)}
$$

proving the Lemma.

Proof of Theorem 2.1. Theorem 2.1 follows directly from Lemma 2.2 and Lemma 2.3 by standard approximation procedure. Namely, for $\gamma \in$ $W^{1, \infty}\left(\mathbb{R}^{n}\right)$ as before we have

$$
\begin{aligned}
S_{\rho} T_{\rho}-I= & \left(S_{\rho}\left(\gamma_{\varepsilon}\right) T_{\rho}\left(\gamma_{\varepsilon}\right)-I\right) \\
& -\left(S_{\rho}\left(\gamma_{\varepsilon}\right)-S_{\rho}(\gamma)\right) T_{\rho}\left(\gamma_{\varepsilon}\right)-S_{\rho}(\gamma)\left(T_{\rho}\left(\gamma_{\varepsilon}\right)-T_{\rho}(\gamma)\right)
\end{aligned}
$$

Since $\left\|\gamma_{\varepsilon}\right\|_{C^{2}}=O\left(\varepsilon^{-2}\right)$ we obtain from Lemma 2.2

$$
\left\|S_{\rho}\left(\gamma_{\varepsilon}\right) T_{\rho}\left(\gamma_{\varepsilon}\right)-I\right\|_{L_{\delta+1}^{2} \rightarrow L_{\delta+1}^{2}} \leq \frac{C\left\|\gamma_{\varepsilon}\right\|_{C^{2}}}{|\rho|}=O\left(\frac{1}{|\rho| \varepsilon^{2}}\right) .
$$


Now by Proposition 2.2

$$
\left\|S_{\rho}(\tilde{\gamma})\right\|_{L_{\delta+1}^{2} \rightarrow L_{\delta+1}^{2}}+\left\|T_{\rho}(\tilde{\gamma})\right\|_{L_{\delta+1}^{2} \rightarrow L_{\delta+1}^{2}} \leq C\|\nabla \log \tilde{\gamma}\|_{L^{\infty}\left(\mathbb{R}^{n}\right)}
$$

holds for any conductivity $\tilde{\gamma} \in W^{1, \infty}\left(\mathbb{R}^{n}\right)$. Therefore by taking $\varepsilon=|\rho|^{-1 / 4}$ and $|\rho|$ large we obtain from Lemma 2.3 and (2.27) the desired conclusion (2.19).

Next we study the behavior of $\omega_{1}$ as $\varepsilon \rightarrow 0$ and $|\rho| \rightarrow \infty$. By Theorem 2.1 and (2.12) we can define

$$
\omega_{1}(x, \varepsilon, \rho)=-\Delta_{\rho}^{-1} S_{\rho} f_{\varepsilon}+h_{\rho}
$$

where

$$
h_{\rho}=\Delta_{\rho}^{-1} C_{\rho} f_{\varepsilon}
$$

with $C_{\rho}=T_{\rho}^{-1}-S_{\rho}$.

\section{Lemma 2.4}

$$
\lim _{|\rho| \rightarrow \infty}\left\|h_{\rho}\right\|_{H_{\delta}^{1}\left(\mathbb{R}^{n}\right)}=0
$$

Proof. By Theorem 2.1 we have that

$$
\left\|T_{\rho}^{-1}\right\|_{L_{\delta+1}^{2} \rightarrow L_{\delta+1}^{2}} \leq C
$$

with $C$ independent of $\rho$. By definition

$$
T_{\rho} \Delta_{\rho} \omega_{1}=-f_{\varepsilon}
$$

and

$$
\Delta_{\rho} \omega_{1}=-S_{\rho} f_{\varepsilon}+C_{\rho} f_{\varepsilon} .
$$

Therefore by Theorem 2.1

$$
\left\|\Delta_{\rho} \omega_{1}+S_{\rho} f_{\varepsilon}\right\|_{L_{\delta+1}^{2} \rightarrow L_{\delta+1}^{2}} \rightarrow 0 \text { as } \rho \rightarrow \infty .
$$

This implies that

$$
A:=\left\|\nabla \omega_{1}+\Delta_{\rho}^{-1} \nabla S_{\rho} f_{\varepsilon}\right\|_{L_{\delta}^{2} \rightarrow L_{\delta}^{2}} \rightarrow 0 \text { as }|\rho| \rightarrow \infty
$$

since

$$
A=\left\|\nabla \Delta_{\rho}^{-1}\left(\Delta_{\rho} \omega_{1}+S_{\rho} f_{\varepsilon}\right)\right\|_{L_{\delta}^{2} \rightarrow L_{\delta}^{2}} \leq C\left\|\Delta_{\rho} \omega_{1}-S_{\rho} f_{\varepsilon}\right\|_{L_{\delta+1}^{2} \rightarrow L_{\delta+1}^{2}}
$$


Proof of Theorem 1.1. The choice $\omega_{0}(x, \rho)=\omega_{0}(x, \varepsilon)$ and $\omega_{1}(x, \rho)=$ $\omega_{1}(x, \varepsilon, \rho)$, with say $\varepsilon=|\rho|^{-\alpha}$ for any $0<\alpha \leq 1$ will lead to the desired result. Indeed, by Proposition 2.2 and by (2.16)

$$
\left\|f_{\varepsilon}\right\| \leq C\left(\varepsilon^{-1}+|\rho|\right) o(\varepsilon) .
$$

On the other hand by Lemma 2.3

$$
\left\|\omega_{1}\right\|_{L_{\delta}^{2}} \leq\left\|\Delta_{\rho}^{-1} S_{\rho} f_{\varepsilon}\right\|_{L_{\delta}^{2}}+\left\|\Delta_{\rho} h_{\rho}\right\|_{L_{\delta}^{2}} \leq \frac{C}{|\rho|}\left\|f_{\varepsilon}\right\|
$$

which proves that

$$
\left\|\omega_{1}\right\|_{L_{\delta}^{2}} \stackrel{|\rho| \rightarrow \infty}{\longrightarrow} 0 .
$$

This together with Lemma 2.1 prove Theorem 1.1.

\section{The uniqueness proof}

In this section we prove Theorem 1.2. We shall use the following identity

Lemma 3.1 Let $\gamma \in W^{1, \infty}(\Omega)$, $\gamma$ strictly positive on $\bar{\Omega}$ and $a=\sqrt{\gamma}$. Let $u \in H^{1}(\Omega)$ be a solution of $\operatorname{div}(\gamma \nabla \mathrm{u})=0$. Let $v \in H^{1}(\Omega)$. Then

$$
\int_{\Omega}(\nabla a \cdot \nabla(u v)-\nabla(a u) \cdot \nabla v) d x=-\int_{\partial \Omega} a v \frac{\partial u}{\partial \nu} d S
$$

where $d S$ denotes surface measure on $\partial \Omega$.

Proof. By the divergence theorem we have

$$
\int_{\Omega} a^{-1} \operatorname{div}\left(a^{2} \nabla u\right) v d x=-\int_{\Omega} a^{2} \nabla u \cdot \nabla\left(\frac{v}{a}\right) d x-\int_{\partial \Omega} a \frac{\partial u}{\partial \nu} v d S .
$$

Therefore

$$
\int_{\Omega} a^{2} \nabla u \cdot \nabla\left(\frac{v}{a}\right) d x=-\int_{\partial \Omega} a \frac{\partial u}{\partial \nu} v d S
$$

Now setting $w=a u$ and using that $\nabla a^{-1}=-a^{-2} \nabla a$ we obtain

$$
\begin{aligned}
\int_{\Omega} a^{2} \nabla & \left(a^{-1} w\right) \cdot \nabla\left(a^{-1} v\right) d x \\
& =\int_{\Omega}\left(\nabla w \cdot \nabla v-\nabla a \cdot\left(w \nabla\left(a^{-1} v\right)+v \nabla\left(a^{-1} w\right)\right)\right) d x \\
& =\int_{\Omega} \nabla w \cdot \nabla v-\nabla a \cdot \nabla\left(a^{-1} w v\right) d x \\
& =\int_{\Omega} \nabla(a u) \cdot \nabla v-\nabla a \cdot \nabla(u v) d x
\end{aligned}
$$

proving the Lemma. 
The right-hand side of (3.1) is determined by the DN map if we know $v_{\mid \partial \Omega}$. This is a consequence of the following two Lemmas.

Lemma $3.2[1,6,14]$ Let $\gamma_{i} \in W^{1, \infty}(\Omega)$ conductivities $i=1,2$ with $\Lambda_{\gamma_{1}}=$ $\Lambda_{\gamma_{2}}$. Then

$$
\gamma_{\left.\right|_{\partial \Omega}}=\left.\gamma_{2}\right|_{\partial \Omega} \quad \text { and } \quad\left(\frac{\partial \gamma_{1}}{\partial \nu}\right)_{\left.\right|_{\partial \Omega}}=\left.\left(\frac{\partial \gamma_{2}}{\partial \nu}\right)\right|_{\partial \Omega} .
$$

Lemma 3.3 [13] Assume $\gamma_{i} \in W^{1, \infty}(\Omega)$ conductivities $i=1,2$ with $\Lambda_{\gamma_{1}}=$ $\Lambda_{\gamma_{2}}$. Extend $\gamma_{i} \in W^{1, \infty}\left(\mathbb{R}^{n}\right)$ with $\gamma_{1}=\gamma_{2}$ in $\Omega^{c}$ and $\gamma_{i}=1$ outside a large ball. Let $u_{i}, i=1,2$ be the complex geometrical solutions as in Theorem 1.1. Then

$$
u_{1}=u_{2} \quad \text { in } \quad \Omega^{c} .
$$

We include the proof here for the sake of completeness.

Proof. Let $z \in H^{1}(\Omega)$ be the solution of

$$
\begin{aligned}
\operatorname{div}\left(\gamma_{1} \nabla z\right) & =0 \quad \text { on } \quad \Omega \\
z_{\left.\right|_{\partial \Omega}} & =u_{\left.2\right|_{\partial \Omega} .}
\end{aligned}
$$

By the assumption $\Lambda_{\gamma_{1}}=\Lambda_{\gamma_{2}}$, we have

$$
\left(\gamma_{1} \frac{\partial z}{\partial \nu}\right)_{\partial \Omega}=\left(\gamma_{2} \frac{\partial u_{2}}{\partial \nu}\right)_{\partial \Omega},
$$

and therefore, since $\gamma_{1}$ equals $\gamma_{2}$ on the boundary,

$$
\left(\frac{\partial z}{\partial \nu}\right)_{\partial \Omega}=\left.\left(\frac{\partial u_{2}}{\partial \nu}\right)\right|_{\partial \Omega} .
$$

Then $z$ solves $\operatorname{div}\left(\gamma_{1} \nabla z\right)=0$ in $\mathbb{R}^{n}$ if we extend $z=u_{2}$ on $\Omega^{c}$. Since $z$ has the same asymptotic behavior as $u_{1}$ we have from the uniqueness of the solutions in Theorem 1.1 that $z=u_{1}$ and therefore $u_{1}=u_{2}$ in $\Omega^{c}$.

Combining Lemmas 3.1-3.3 we arrive at the following:

Proposition 3.1 Let $\gamma_{i} \in W^{1, \infty}(\Omega)$ conductivities with $\Lambda_{\gamma_{1}}=\Lambda_{\gamma_{2}}$ and let $a_{i}=\gamma_{i}^{1 / 2}$. Then

$$
\begin{aligned}
\int_{\Omega}\left(\nabla a_{1} \cdot \nabla\left(u_{1} v\right)\right. & \left.-\nabla a_{2} \cdot \nabla\left(u_{2} v\right)\right) d x \\
& -\int_{\Omega}\left(\nabla\left(a_{1} u_{1}\right) \cdot \nabla v-\nabla\left(a_{2} u_{2}\right) \cdot \nabla v\right) d x=0,
\end{aligned}
$$

for any $v \in H^{1}(\Omega)$ and $u_{i} \in H^{1}(\Omega)$ solution of $\operatorname{div}\left(\gamma_{i} \nabla u_{i}\right)=0$ on $\Omega, i=1,2$. 
The next lemma is the only place where the assumption $\gamma \in W^{3 / 2, \infty}(\Omega)$ is needed.

Lemma 3.4 Let $\gamma \in W^{3 / 2, \infty}\left(\mathbb{R}^{n}\right)$, $\gamma$ strictly positive and equal to one outside a large ball. Then for $\omega_{1}(x, \rho)=\omega_{1}(x, \varepsilon, \rho)$, as in $(2.28), \varepsilon=|\rho|^{-1}$ we have

$$
\int e^{i x \cdot \xi} \nabla \gamma^{1 / 2} \cdot \nabla \omega_{1} d x \stackrel{|\rho| \rightarrow \infty}{\longrightarrow} 0
$$

Proof. The statement (3.6) is equivalent to

$$
\int e^{i x \cdot \xi} \gamma^{1 / 2} A \cdot \nabla \omega_{1} d x \stackrel{|\rho| \rightarrow \infty}{\longrightarrow} 0
$$

Using (2.12), (2.16) and (2.19), (3.7) follows if we show

$$
\int e^{i x \cdot \xi} \gamma^{-1 / 2} A \cdot \nabla \Delta_{\rho}^{-1} f_{\varepsilon} d x \stackrel{|\rho| \rightarrow \infty}{\longrightarrow} 0
$$

and

$$
\int e^{i x \cdot \xi} \gamma^{-1 / 2} A \cdot \nabla \Delta_{\rho}^{-1} \gamma^{-1 / 2} A \cdot \nabla_{\rho} \Delta_{\rho}^{-1} \gamma^{1 / 2} f_{\varepsilon} d x \stackrel{|\rho| \rightarrow \infty}{\longrightarrow} 0
$$

We know from Lemma 2.1 that $e^{\frac{\varphi_{\varepsilon}}{2}} \rightarrow \gamma^{1 / 2}$ in $H_{\delta}^{1}$ so that it can be easily estimated. The first two terms of $f_{\varepsilon}$ involve $\Delta \varphi_{\varepsilon}$ and $\nabla \varphi_{\varepsilon}$. Recall from Proposition 2. that $\nabla \varphi_{\varepsilon}=A_{\varepsilon}$ and therefore $\Delta \varphi_{\varepsilon}=\operatorname{div} \mathrm{A}_{\varepsilon}$. Below we will see that the choice $\varepsilon \simeq|\rho|^{-1}$ is the only possibility to obtain (3.6). Accordingly in (2.14) the terms $\nabla \cdot A_{\varepsilon}=\Delta \varphi_{\varepsilon}$ and $\left(A-A_{\varepsilon}\right) \cdot \rho$ behave both as $\varepsilon^{-1 / 2} O(\varepsilon)$. The other two terms are bounded and cause no trouble. We will give detailed estimates for the term involving $\left(A-A_{\varepsilon}\right) \cdot \rho$. The same reasoning works for the other terms, as well. To this end we notice that for (3.9) we need to show

$C_{\varepsilon, \rho}:=\int e^{i x \cdot \xi} \gamma^{-1 / 2}\left(A-A_{\varepsilon}\right) \cdot \nabla \Delta_{\rho}^{-1} \gamma^{-1 / 2} A \cdot \nabla_{\rho} \Delta_{\rho}^{-1} \gamma^{1 / 2}\left(A-A_{\varepsilon}\right) \cdot \rho d x \stackrel{|\rho| \rightarrow \infty}{\longrightarrow} 0$.

and

$$
D_{\varepsilon, \rho}:=\int e^{i x \cdot \xi} \gamma^{-1 / 2} A_{\varepsilon} \cdot \nabla \Delta_{\rho}^{-1} \gamma^{-1 / 2} A \cdot \nabla_{\rho} \Delta_{\rho}^{-1} \gamma^{1 / 2}\left(A-A_{\varepsilon}\right) \cdot \rho d x \stackrel{|\rho| \rightarrow \infty}{\longrightarrow} 0 .
$$

Since $\nabla \Delta_{\rho}^{-1}$ and $\nabla_{\rho} \Delta_{\rho}^{-1}$ are both bounded operators from $L_{\delta+1}^{2} \longrightarrow L_{\delta}^{2}$ we have by choosing $\varepsilon=|\rho|^{-1}$

$$
C_{\varepsilon, \rho}=\varepsilon O(\varepsilon)|\rho| \stackrel{|\rho| \rightarrow \infty}{\longrightarrow} 0 .
$$


On the other hand, since

$$
\left\|\nabla\left(e^{i x \cdot \xi} \gamma^{-1 / 2} A_{\varepsilon}\right)\right\|_{L^{2}}=\varepsilon^{-1 / 2} o(\varepsilon)
$$

the integration by parts gives

$$
D_{\varepsilon, \rho}=\varepsilon^{-1 / 2} \varepsilon^{1 / 2} O(\varepsilon) \stackrel{|\rho| \rightarrow \infty}{\longrightarrow} 0 .
$$

proving (3.9) concerning this term. The proof of (3.8) is similar but easier.

Note that the above proof uses the result of Theorem 2.1. The proof of this theorem uses the choice $\varepsilon=|\rho|^{-1 / 4}$ but the statement of the theorem is independent of $\varepsilon$.

Proof of Theorem 1.2. We now extend $\gamma_{i} \in W^{3 / 2, \infty}\left(\mathbb{R}^{n}\right)$ with $\gamma_{1}=\gamma_{2}$ on $\Omega^{c}, \gamma_{i}=1$ outside a large ball $i=1,2$ and $\gamma_{i}$ strictly positive.

Let $\xi \in \mathbb{R}^{n}$. We choose $\rho_{1} \in \mathbb{C}^{n}, \rho_{1} \cdot \rho_{1}=0,\left|\rho_{1}\right|$ large enough and $\rho_{2} \in \mathbb{C}^{n}, \rho_{2} \cdot \rho_{2}=0$ so that $\rho_{1}+\rho_{2}=i \xi$. Let

$$
u_{i}=e^{x \cdot \rho_{1}}\left(\omega_{0}^{(i)}+\omega_{1}^{(i)}\right), \quad i=1,2,
$$

as in Theorem 1.1 with $\gamma$ replaced by $\gamma_{i}, i=1,2$. Let

$$
v=e^{x \cdot \rho_{2}} .
$$

Now we substitute (3.10) and (3.11) in (3.5). We note that by Lemmas 3.2 and 3.3 we can replace the integration over $\Omega$ on (3.5) by integration over $\mathbb{R}^{n}$.

By taking the limit as $|\rho| \rightarrow \infty$ and $\varepsilon \rightarrow 0$ we can replace by Lemmas 2.1 and $3.3 u_{1}$ by $e^{x \cdot \rho_{1}} \gamma_{1}^{-1 / 2}$ and $u_{2}$ by $e^{x \cdot \rho_{2}} \gamma_{2}^{-1 / 2}$ in (3.5). We obtain

$$
\int_{\mathbb{R}^{n}}\left(\nabla \gamma_{1}^{1 / 2} \cdot \nabla\left(e^{i x \cdot \xi} \gamma_{1}^{-1 / 2}\right)-\nabla \gamma_{2}^{1 / 2} \cdot \nabla\left(e^{i x \cdot \xi} \gamma_{2}^{-1 / 2}\right)\right) d x=0
$$

This leads to

$$
\begin{aligned}
\frac{i}{2} \xi \cdot \int & e^{i x \cdot \xi}\left(\nabla \log \gamma_{1}-\nabla \log \gamma_{2}\right) d x \\
& -\frac{1}{4} \int e^{i x \cdot \xi}\left(\left(\nabla \log \gamma_{1}\right)^{2}-\left(\nabla \log \gamma_{2}\right)^{2}\right) d x=0 .
\end{aligned}
$$

Then by using the Fourier inversion formula we obtain in the sense of distributions

$$
\Delta\left(\log \gamma_{1}-\log \gamma_{2}\right)+\frac{1}{2}\left(\nabla \log \gamma_{1}+\nabla \log \gamma_{2}\right) \cdot \nabla\left(\log \gamma_{1}-\log \gamma_{2}\right)=0 .
$$

Since $\gamma_{\left.\right|_{\partial \Omega \Omega}}=\gamma_{\left.2\right|_{\partial \Omega}}$, by the uniqueness of the solutions of the Dirichlet problem we conclude that $\gamma_{1}=\gamma_{2}$ ending the proof of Theorem 1.2. 


\section{References}

[1] Alessandrini, G.: Singular solutions of elliptic equations and the determination of conductivity by boundary measurements. J. Diff. Equations 84 (1990), 252-272.

[2] Brown, R.: Global uniqueness in the impedance imaging problem for less regular conductivities. SIAM J. Math. Anal. 27 (1996), 1049-1056.

[3] Brown, R. and Uhlmann, G.: Uniqueness in the inverse conductivity problem for nonsmooth conductivities in two dimensions. Comm. PDE 22 (1997), 1009-1027.

[4] Calderón, A. P.: On an inverse boundary value problem. Seminar on Numerical Analysis and its Applications to Continuum Physics, 65-73. Soc. Brasileira de Matemática, 1980.

[5] Colton, D. And PÄrvärinta, L.: The uniqueness of a solution to an inverse scattering problem for electromagnetic waves. Arch. Rational Mech. Anal. 119 (1992), 59-70.

[6] Kohn, R. And Vogelius, M.: Determining conductivity by boundary measurements. Comm. Pure Appl. Math. 37 (1984), 289-298.

[7] Kohn, R. And Vogelius, M.: Determining conductivity by boundary measurements II. Interior results. Comm. Pure Appl. Math. 38 (1985), 643-667.

[8] Nachman, A.: Global uniqueness for a two-dimensional inverse boundary value problem. Ann. of Math. 143 (1996), 71-96.

[9] Nakamura, G., Sun, Z. and Uhlmann, G.: Global identifiability for an inverse problem for the Schrödinger equation in a magnetic field. Math. Ann. 303 (1995), 377-388.

[10] Panchenko, A.: An inverse problem for the magnetic Schrödinger equation and quasi-exponential solutions of nonsmooth partial differential equations. Inverse Problems 18 (2002), 1421-1439.

[11] Sun, Z.: An inverse boundary value problem for Schrödinger operator with vector potential. Trans. Amer. Math. Soc. 338 (1993), 953-959.

[12] Sylvester, J. and Uhlmann, G.: A uniqueness theorem for an inverse boundary value problem in electrical prospection. Comm. Pure Appl. Math. 39 (1986), 91-112.

[13] Sylvester, J. and Uhlmann, G.: A global uniqueness theorem for an inverse boundary value problem. Ann. of Math. 125 (1987), 153-169.

[14] Sylvester, J. and Uhlmann, G.: Inverse boundary value problems at the boundary-continuous dependence. Comm. Pure Appl. Math 41 (1988), $197-221$. 
[15] Tolmasky, C.: Exponentially growing solutions for nonsmooth first order perturbations of the Laplacian. SIAM J. Math. Anal. 29 (1998), 116-133.

[16] Uhlmann, G.: Developments in inverse problems since Calderón's foundational paper. Harmonic analysis and partial differential equations (Chicago, IL, 1996), 295-345 (M. Christ, C. Kenig, C. Sadosky editors). Chicago Lectures in Math., Univ. Chicago Press, 1999.

Recibido: 7 de mayo de 2001

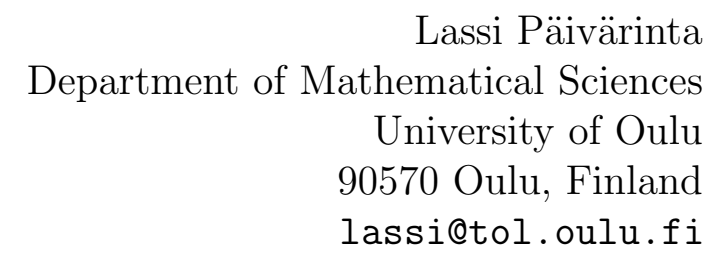

Alexander Panchenko Department of Mathematics Pennsylvania State University University Park, PA 16802, USA panchenk@math.psu.edu

Gunther Uhlmann Department of Mathematics University of Washington Seattle, WA 98195, USA gunther@math . washington . edu 